\title{
Capacity Planning of a Perinatal Network with Generalised Loss Network Model with Overflow
}

\author{
Md Asaduzzaman* \\ Institute of Statistical Research and Training (ISRT), University of Dhaka, Dhaka 1000, \\ Bangladesh \\ Thierry J. Chaussalet \\ Department of Business Information Systems, School of Electronics and Computer \\ Science, University of Westminster, 115 New Cavendish Street, London W1W 6UW, UK
}

\begin{abstract}
Recent literature shows that the arrival and discharge processes in hospital intensive care units do not satisfy the Markovian property, that is, interarrival times and length of stay tend to have a long tail. In this paper we develop a generalised loss network framework for capacity planning of a perinatal network in the UK. Decomposing the network by hospitals, each unit is analysed with a GI/G/c/0 overflow loss network model. A two-moment approximation is performed to obtain the steady state solution of the GI/G/c/0 loss systems, and expressions for rejection probability and overflow probability have been derived. Using the model framework, the number of required cots can be estimated based on the rejection probability at each level of care of the neonatal units in a network. The generalisation ensures that the model can be applied to any perinatal network for renewal arrival and discharge
\end{abstract}

\footnotetext{
${ }^{*}$ Corresponding author

Email addresses: asad@isrt.ac.bd (Md Asaduzzaman), chausst@wmin.ac.uk (Thierry J. Chaussalet) 
processes.

Key words: OR in Health Care, Bed planning, ICU rejection probability, Queueing network, Overflow model, Non-Markovian system 2000 MSC: 90B22, 60K25

\section{Introduction}

In most of the developed world neonatal care has been organised into networks of cooperating hospitals (units) in order to provide better and more efficient care for the local population. A neonatal or perinatal network in the UK offers all ranges of neonatal care referred to as intensive, high dependency and special care through level 1 to level 3 units. Recent studies show that perinatal networks in the UK have been struggling with severe capacity crisis (Bliss, 2007; National Audit Office, 2007). Expanding capacity by number of beds in the unit, in general, is not an option since neonatal care is an unusually expensive therapy. Reducing capacity is not an option either, as this would risk sick neonates being denied admission to the unit or released prematurely. Consequently, determining cot capacity has become a major concern for perinatal network managers in the UK. We have addressed this issue in several papers (Asaduzzaman and Chaussalet, 2008; Asaduzzaman et al., 2010a,b; Asaduzzaman, 2010; Asaduzzaman and Chaussalet, 2011). How-

ever, as noted by Asaduzzaman et al. (2010a) and Asaduzzaman (2010) the mean is much lower than standard deviation for interarrival times and length of stay (LOS). Clearly this is a violation of the property of the exponential distribution, and hence of the Markovian assumption. Asaduzzaman (2010) has reported that the graphs and diagrams of the interarrival and LOS show 
a non-exponential pattern, and that a mixture of distributions would provide a better fit. Moreover, several mathematical (Vasilakis and Marshall, 2005; Griffiths et al., 2006; Asaduzzaman, 2010) and medical (Weissman, 1997, 2000; Rapoport et al., 2003) studies also show that the length of stay is heavily skewed, i.e. has a long tail, in ICU in particular.

Queueing models having zero buffer also referred to as 'loss models' (./././0) have been widely applied in hospital systems and intensive care in particular (e.g., Van Dijk and Kortbeek, 2009; Litvak et al., 2008; Asaduzzaman et al., 2010a,b; Asaduzzaman and Chaussalet, 2011). Van Dijk and Kortbeek (2009) proposed an M/M/c/0 loss model for capacity management in an Operating Theatre-Intensive Care Unit. Litvak et al. (2008) developed an overflow model with loss framework for capacity planning in intensive care units while Asaduzzaman et al. (2010a,b) developed a loss network model for a neonatal unit, and extended the model framework to a perinatal network in Asaduzzaman and Chaussalet (2011). These models assume that interarrival times and length of stay follow exponential distributions.

Queueing models with exponential inter-arrival and service times are easiest to study, since such processes are Markov chains. However, length of stay distribution in intensive care may be highly skewed (Griffiths et al., 2006). Performance measures of a queueing system with non-zero buffer are insensitive to service time distribution provided that the arrival process is Poisson (Kelly, 1979). This insensitivity property is, in general, no longer valid in the case of zero buffer or loss systems (Klimenok et al., 2005).

Many approaches have been found towards generalising such processes since Erlang introduced the $\mathrm{M} / \mathrm{M} / \mathrm{c} / 0$ model for a simple telephone network 
and derived the well-known loss formula that carries his name in 1917 (Kelly, 1991; Whitt, 2004). Takács $(1956,1962)$ considered the loss system with general arrival pattern $(\mathrm{GI} / \mathrm{M} / \mathrm{c} / 0)$ through Laplace transform. Recently there has been a growing interest in loss systems where both arrival and service patterns are generalised $(\mathrm{GI} / \mathrm{G} / \mathrm{c} / 0)$. The theoretical investigation of the $\mathrm{GI} / \mathrm{G} / \mathrm{c} / 0$ loss model through the theory of random point processes has attracted many researchers. Brandt and Lisek (1980) gave a method for approximating the GI/GI/c/0 queue by means of the GI/GI/ $\infty$ queue, while Whitt (1984) applied a similar approximation under heavy traffic. Franken et al. (1982) examined the continuity property of the model, and established an equivalence between arrival and departure probability. Miyazawa and Tijms (1993) gave an approximation method for the batch-arrival GI ${ }^{[x]} / \mathrm{G} / \mathrm{c} / \mathrm{N}$ queue which is applicable when the traffic intensity is less than one. The $\mathrm{M} / \mathrm{G} / \mathrm{c} / \mathrm{N}$ and the $\mathrm{GI} / \mathrm{G} / \mathrm{c} / \mathrm{N}$ queue have also been studied widely; for a comparison of methods, see Kimura (2000). Although many studies have been found in the literature, no simple expression for the steady state distribution is available for a GI/G/c/0 system. Hsin and Van de Liefvoort (1996) provided the exact solution for the GI/GI/c/0 system expressing the interarrival and service time by matrix exponential distribution. The method is computationally intensive and often includes imaginary components in the expression (which are unrealistic). Diffusion approximations, which require complicated Laplace transforms have also been used for analysing GI/G/c/N queues (e.g., Kimura, 2003; Whitt, 2004). Kim and Chae (2003) derived a transform-free expression for the analysis of the GI/G/1/N queue through the decomposed Little's formula. A two-moment approximation was proposed to 
estimate the steady state queue length distribution. Using the same approximation, Choi et al. (2005) extended the system for the multi-server finite buffer queue based on the system equations derived by Franken et al. (1982). Atkinson (2009) developed a heuristic approach for the numerical analysis of GI/G/c/0 queueing systems with examples of the two-phase Coxian distribution. Recently, Izady and Worthington (2011) performed an approximate analysis of time-dependent loss queues and networks of loss queues where general service time distributions are considered.

In this paper we derive a generalised loss network model with overflow for a network of neonatal hospitals extending the results obtained by Franken et al. (1982). Since some model parameters cannot be computed practically, a two-moment based approximation method is applied for the steady state analysis as proposed by Kim and Chae (2003). The model is then applied to the north central London Perinatal network, one of the busiest network in the UK. Data obtained from each hospital (neonatal unit) of the network have been used to check the performance of the model. The rest of the paper is organised as follows: in the next section we first discuss a typical perinatal network and then develop a generalised loss model with overflow for the network. The steady state distribution and expression for rejection and overflow probabilities have been derived for each level of care of the neonatal units. Application of the model and numerical results are presented in Section 4 . 


\section{Structure of a perinatal network}

A perinatal network in the UK is organised through level 1, level 2 and level 3 units. Figure 1 shows a typical perinatal network in the UK. Level 1 units consist of a special care baby unit (SCBU). It provides only special care which is the least intensive and most common type of care. In these units, neonates may be fed through a tube, supplied with extra oxygen or treated with ultraviolet light for jaundice. Figure 2 shows the typical patient flow in a level 1 unit. A level 1 unit may also have an intensive therapy unit (ITU) which provides short-term intensive care to neonates, and the unit may then be referred to as 'level 1 unit with ITU'. Figure 3 shows the structure of a level 1 unit with ITU. Level 2 units consist of a SCBU and a HDU where neonates can receive high dependency care such as breathing via continuous positive airway pressure or intravenous feeding. These units may also provide short-term intensive care. A level 3 unit provides all ranges of neonatal care and consists of an SCBU, an HDU and an NICU where neonates will often be on a ventilator and need constant care to be kept alive. Level 2 and level 3 units may also have some transitional care (TC) cots, which may be used to tackle overflow and rejection from SCBU. Although level 2 and level 3 units have similar structures level 2 units might not have sufficient clinician support for the NICU. NICU are HDU are often merged in level 2 and level 3 units for higher utilisation of cots. In level 2 or level 3 units, NICU-HDU neonates are sometimes initially cared at SCBU when all NICU cots are occupied. Similarly SCBU neonates are cared at NICU-HDU or TC, depending upon the availability of cots, staff and circumstances. This temporary care is provided by staffing a cot with appropriate nurse and 


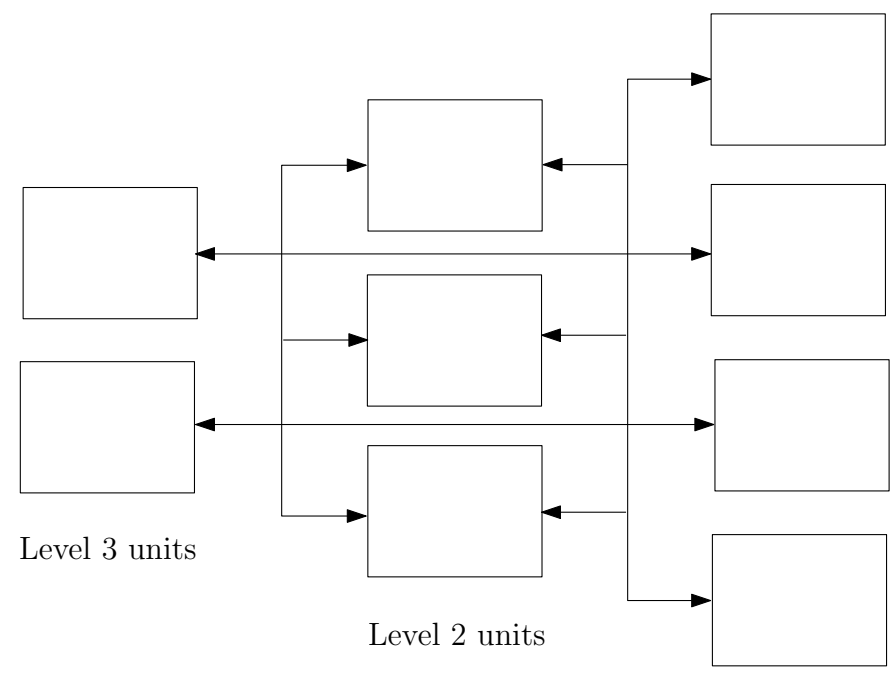

Level 1 units

Figure 1: Topology of a typical perinatal network. The arrows indicate forward and backward transfers between units.

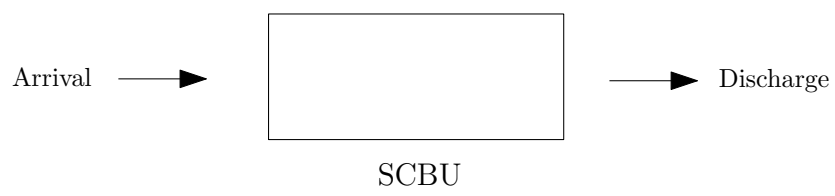

Figure 2: Sub-network model for a level 1 unit.

equipment resources, and will be referred to as 'overflow'. Rejection occurs only when all cots are occupied; in such cases neonates are transferred to another neonatal unit. Patient flows in a typical level 3 or level 2 unit are depicted in Figure 4. Unlike for level 3/level 2 units, overflow does not occur in level 1 units with ITU.

The underlying admission, discharge and transfer policies of a perinatal network are described below.

1. All mothers expecting birth $<27$ week of gestational age or all neonates 


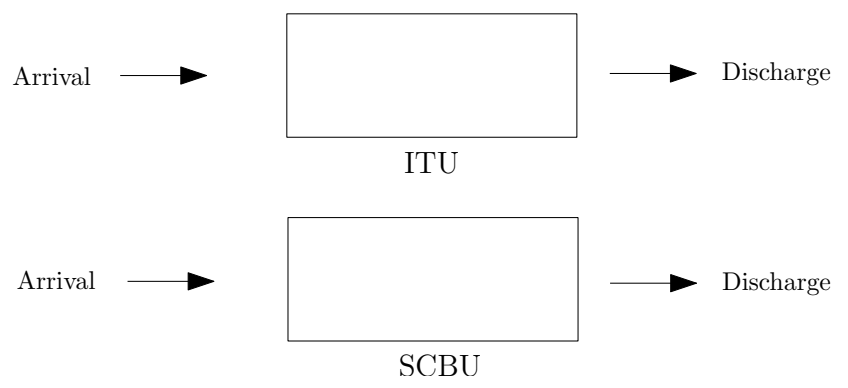

Figure 3: Sub-network model for a level 1 unit with ITU.

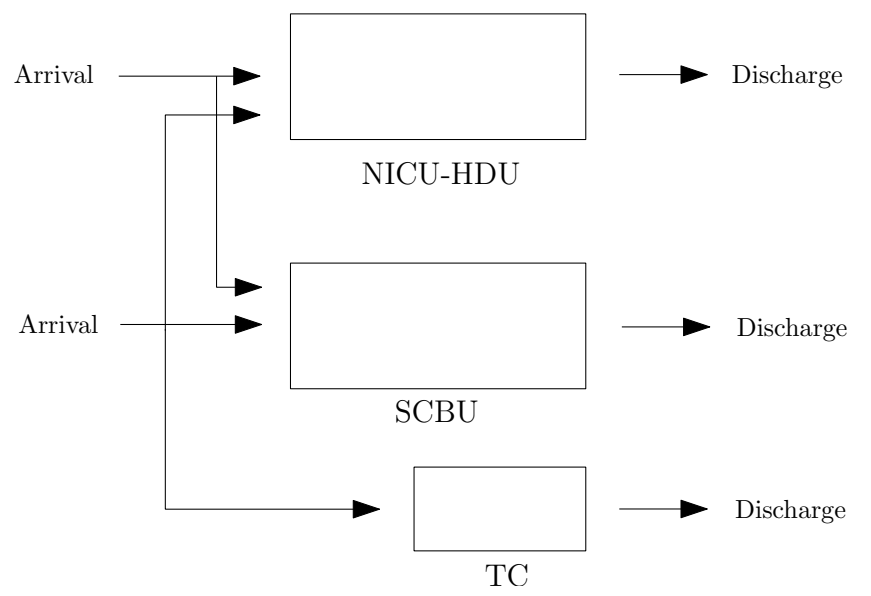

Figure 4: Sub-network model for a level 3/level 2 unit.

with $<27$ week of gestational age are transferred to a level 3 unit.

2. All mothers expecting birth $\geq 27$ but $<34$ week of gestational age or all neonates of the same gestational age are transferred to a level 2 unit depending upon the booked place of delivery.

3. All neonatal units accept neonates for special care booked at the same unit.

4. Neonates admitted into units other than their booked place of delivery are transferred back to their respective neonatal unit receiving after the required level of care. 
Now we shall develop a generalised loss network framework for a perinatal network with level 1, level 2 and level 3 units. To obtain the steady state behavior of the network, we first decompose the whole network into a set of subnetworks (i.e., each neonatal units) due to higher dimensionality, then we derive the steady state solution and expression of rejection probability for each of the units. When analysing a particular sub-network in isolation, back transfers are combined with new arrivals to specifically take into account the dependencies between units. Cot capacity for the neonatal units may be determined based on the rejection probabilities at each level of care and overflow to temporary care of the units.

\section{Mathematical model formulation}

\subsection{Model for a level 1 unit}

A level 1 unit consists typically of a SCBU. Therefore, assuming no waiting space and first come first served (FCFS) discipline, a level 1 unit can be modelled as a GI/G/c/0 system. Let the inter-arrival times and length of stay of neonates be i.i.d. random variables denoted by $A$ and $L$, respectively. Also the length of stay is independent of the arrival process. Define

$$
m_{A}=\mathbb{E}(A)=\frac{1}{\lambda}, \quad m_{L}=\mathbb{E}(L)=\frac{1}{\mu} .
$$

Let $N$ denotes the number of neonates in the system at an arbitrary time, $N^{a}$ denotes the number of neonates (arriving) who find the system is in steady state with $N$ neonates, and $N^{d}$ denotes the number of neonates discharged from the system in steady state with $N$ neonates. Let $c$ be the number of cots at the SCBU. For $0 \leq n \leq c$, let

$$
\pi(n)=\mathbb{P}(N=n)
$$




$$
\pi^{a}(n)=\mathbb{P}\left(N^{a}=n\right), \quad \quad \pi^{d}(n)=\mathbb{P}\left(N^{d}=n\right),
$$

and

$$
m_{A, n}^{d}=\mathbb{E}\left(A_{n}^{d}\right), \quad m_{L, n}^{a}=\mathbb{E}\left(L_{n}^{a}\right), \quad m_{L, n}^{d}=\mathbb{E}\left(L_{n}^{d}\right),
$$

where $A_{n}^{d}$ is the remaining inter-arrival time at the discharge instant of a neonate who leaves behind $n$ neonates in the systems, $L_{n}^{a}\left(L_{n}^{d}\right)$ is the remaining length of stay of a randomly chosen occupied cot at the arrival (discharge) instant of a neonate who finds (leaves behind) $n$ neonates in the system.

Let $m_{A, n}^{a}$ and $m_{L, n}^{* a}$ be, respectively, the mean inter-arrival time and the mean length of stay under the condition that the system started at the arrival instant of a neonate when there were $n$ neonates in the system. Clearly,

$$
m_{A, n}^{a}=m_{A}, \quad m_{L, n}^{* a}=m_{L} .
$$

From the definitions, we obtain

$$
m_{A, c}^{d}=m_{A}, \quad m_{L, c}^{a}=m_{L, c}^{d} .
$$

We set

$$
m_{A,-1}^{a}=0, \quad m_{A,-1}^{d}=0, \quad m_{L, 0}^{a}=0, \quad m_{L, 0}^{d}=0
$$

for convenience. Then the first set of system equations obtained by Franken et al. (1982) for a GI/G/c/0 loss system can be written as

$$
\pi(n)-\lambda m_{A, n-1}^{a} \pi^{a}(n-1)=-\lambda m_{A, n-1}^{d} \pi^{d}(n-1)+\lambda m_{A, n}^{d} \pi^{d}(n), \quad 0 \leq n \leq c .
$$


The second set of system equations can be given by

$$
\begin{aligned}
& n \pi(n)+(n-1) \lambda m_{L, n-1}^{d} \pi^{d}(n-1)-n \lambda m_{L, n}^{d} \pi^{d}(n) \\
= & \lambda m_{L, n-1}^{* a} \pi^{a}(n-1)+(n-1) \lambda m_{L, n-1}^{a} \pi^{a}(n-1)-n \lambda m_{L, n}^{a} \pi^{a}(n), \quad 1 \leq n \leq c-1,
\end{aligned}
$$

and

$c \pi(c)+(c-1) \lambda m_{L, c-1}^{d} \pi^{d}(c-1)=\lambda m_{L, n}^{* a} \pi^{a}(c-1)+(c-1) \lambda m_{L, c-1}^{a} \pi^{a}(c-1)$.

From the first set of system equations for the GI/G/c/0 queue, the following equations can be derived

$$
\pi(0)=\lambda m_{A, 0}^{d} \pi^{d}(0)
$$

and

$$
\pi(n)=\lambda m_{A, n}^{d} \pi^{d}(n)+\lambda m_{A} \pi^{a}(n-1)-\lambda m_{A, n-1}^{d} \pi^{d}(n-1), \quad 1 \leq n \leq c .
$$

From the second set of system equations for the GI/G/c/0 queue, the following equations can be derived

$$
\begin{aligned}
\pi(n)=\frac{1}{n} & {\left[\lambda \pi^{a}(n-1)\left(m_{L}+(n-1) m_{L, n-1}^{a}\right)+\lambda n m_{L, n}^{d} \pi^{d}(n)\right.} \\
& \left.-(n-1) \lambda m_{L, n-1}^{d} \pi^{d}(n-1)-\lambda n m_{L, n}^{a} \pi^{a}(n)\right], \quad 1 \leq n \leq c-1,
\end{aligned}
$$

and

$$
\pi(c)=\frac{1}{c}\left[\lambda \pi^{a}(c-1)\left(m_{L}+(c-1) m_{L, c-1}^{a}\right)-(c-1) \lambda m_{L, c-1}^{d} \pi^{d}(c-1)\right] .
$$

Theorem 1. The steady state distribution for a $G I / G / c / 0$ system is given by

$$
\pi^{a}(n)=\pi^{d}(n)=K^{-1} \prod_{i=0}^{n-1} \frac{\lambda_{i}}{\mu_{i+1}}, \quad 0 \leq n \leq c
$$


and

$$
\pi(n)=\pi^{a}(n) \varphi_{n}=\pi^{d}(n) \varphi_{n}, \quad 0 \leq n \leq c,
$$

where

$$
K=\sum_{n=0}^{c} \prod_{i=0}^{n-1} \frac{\lambda_{i}}{\mu_{i+1}}
$$

and

$$
\begin{aligned}
& \frac{1}{\mu_{i}}=m_{L}-i\left(m_{A}-m_{A, i-1}^{d}\right)+(i-1)\left(m_{L, i-1}^{a}-m_{L, i-1}^{d}\right), \quad 1 \leq i \leq c, \\
& \frac{1}{\lambda_{i}}=\left\{\begin{array}{l}
(i+1)\left(m_{A, i+1}^{d}+m_{L, i+1}^{a}-m_{L, i+1}^{d}\right), \quad 0 \leq i \leq c-2, \\
c m_{A}, \quad i=c-1,
\end{array}\right. \\
& \varphi_{i}=\left\{\begin{array}{l}
\lambda m_{A, 0}^{d}, \quad i=0, \\
\lambda\left[m_{A, i}^{d}+\left(m_{A}-m_{A, i-1}^{d}\right) \mu_{i} / \lambda_{i-1}\right], \quad 1 \leq i \leq c .
\end{array}\right.
\end{aligned}
$$

Proof. The steady state distribution can be obtained by solving the above two sets of system equations. First, we equate equations (1) and (2) with equations (3) and (4) for each $n, 0 \leq n \leq c$. Then using the following well-known rate conservation principle, we solve them simultaneously,

$$
\pi^{a}(n)=\pi^{d}(n)
$$

Hence we obtain equation (5).

In steady state analysis of a GI/G/c/0 system, equations in (6) involve quantities $m_{A, n}^{d}, m_{L, n}^{a}$ and $m_{L, n}^{d}$, which are not easy to compute in general, except for some special cases such as Poisson arrival or exponential length of stay. Therefore, a two moment approximation is used as proposed by Kim and Chae (2003) and Choi et al. (2005) for the steady state distribution of the 
$\mathrm{GI} / \mathrm{G} / \mathrm{c} / 0$ system based on the exact results as derived in equations 5 and 6 . To obtain the approximation, we replace the inter-arrival and length of stay average quantities $m_{A, n}^{d}, m_{L, n}^{a}$ and $m_{L, n}^{d}$ by their corresponding time-average quantities;

$$
\begin{gathered}
m_{A, n}^{d} \approx q_{A}=\frac{\mathbb{E}\left(A^{2}\right)}{2 \mathbb{E}(A)}=\frac{\left(1+c_{A}^{2}\right) m_{A}}{2}, 0 \leq n \leq c-1, \\
m_{L, n}^{a}=m_{L, n}^{d} \approx q_{L}=\frac{\mathbb{E}\left(L^{2}\right)}{2 \mathbb{E}(L)}=\frac{\left(1+c_{L}^{2}\right) m_{L}}{2}, 0 \leq n \leq c-1,
\end{gathered}
$$

where $c_{A}^{2}\left(c_{L}^{2}\right)$ is the squared coefficient of variation of inter-arrival times (length of stay).

Using equations (7) and (8) in equation (5), we obtain the two moment approximation for the steady state distribution

$$
\tilde{\pi}^{a}(n)=\tilde{\pi}^{d}(n)=\tilde{K}^{-1} \prod_{i=0}^{n-1} \frac{\tilde{\lambda}_{i}}{\tilde{\mu}_{i+1}}, \quad 0 \leq n \leq c,
$$

and

$$
\tilde{\pi}(n)=\tilde{\pi}^{a}(n) \tilde{\varphi}_{n}=\tilde{\pi}^{d}(n) \tilde{\varphi}_{n}, \quad 0 \leq n \leq c,
$$

where

$$
\tilde{K}=\sum_{n=0}^{c} \prod_{i=0}^{n-1} \frac{\tilde{\lambda}_{i}}{\tilde{\mu}_{i+1}}
$$

and

$$
\begin{aligned}
& \frac{1}{\tilde{\mu}_{i}}=m_{L}-i\left(m_{A}-q_{A}\right), \quad 1 \leq i \leq c, \\
& \frac{1}{\tilde{\lambda}_{i}}=\left\{\begin{array}{l}
(i+1) q_{A}, \quad 0 \leq i \leq c-2, \\
c m_{A}, \quad i=c-1,
\end{array}\right. \\
& \tilde{\varphi}_{i}=\left\{\begin{array}{l}
\lambda q_{A}, \quad i=0, \\
\lambda\left[q_{A}+\left(m_{A}-q_{A}\right) \tilde{\mu}_{i} / \tilde{\lambda}_{i-1}\right], \quad 1 \leq i \leq c-1, \\
\lambda\left[m_{A}+\left(m_{A}-q_{A}\right) \tilde{\mu}_{i} / \tilde{\lambda}_{i-1}\right], \quad i=c .
\end{array}\right.
\end{aligned}
$$


Therefore, the rejection probability for a level 1 unit is computed as

$$
R=\tilde{\pi}(n) / \sum_{n=0}^{c} \tilde{\pi}(n) .
$$

\subsection{Model for a level 1 neonatal unit with ITU}

In a level 1 unit with ITU (Figure 3), overflow from ITU to SCBU does not occur. The unit can be modelled as two joint GI/G/c/0 systems. Therefore, extending the Theorem 1, the steady state distribution for a level 1 neonatal unit with ITU is given by

$$
\pi^{a}(\mathbf{n})=\pi^{d}(\mathbf{n})=K^{-1} \prod_{i=0}^{\left(n_{1}-1\right)} \prod_{j=0}^{\left(n_{2}-1\right)} \frac{\lambda_{1 i}}{\mu_{1(i+1)}} \cdot \frac{\lambda_{2 j}}{\mu_{2(j+1)}},
$$

and

$$
\pi(\mathbf{n})=\pi^{a}(\mathbf{n}) \varphi_{\mathbf{n}},
$$

where

$$
K=\sum_{n_{1}, n_{2}} \prod_{i=0}^{\left(n_{1}-1\right)} \prod_{j=0}^{\left(n_{2}-1\right)} \frac{\lambda_{1 i}}{\mu_{1(i+1)}} \cdot \frac{\lambda_{2 j}}{\mu_{2(j+1)}} .
$$

The approximate steady state distribution for a level 1 neonatal unit with ITU is given by

$$
\tilde{\pi}^{a}(\mathbf{n})=\tilde{\pi}^{d}(\mathbf{n})=\tilde{K}^{-1} \prod_{i=0}^{\left(n_{1}-1\right)} \prod_{j=0}^{\left(n_{2}-1\right)} \frac{\tilde{\lambda}_{1 i}}{\tilde{\mu}_{1(i+1)}} \cdot \frac{\tilde{\lambda}_{2 j}}{\tilde{\mu}_{2(j+1)}},
$$

and

$$
\tilde{\pi}(\mathbf{n})=\tilde{\pi}^{a}(\mathbf{n}) \tilde{\varphi}_{\mathbf{n}},
$$

where

$$
\tilde{K}=\sum_{n_{1}, n_{2}} \prod_{i=0}^{\left(n_{1}-1\right)} \prod_{j=0}^{\left(n_{2}-1\right)} \frac{\tilde{\lambda}_{1 i}}{\tilde{\mu}_{1(i+1)}} \cdot \frac{\tilde{\lambda}_{2 j}}{\tilde{\mu}_{2(j+1)}} .
$$


and $\tilde{\lambda}_{1 i}, \tilde{\mu}_{1 i}, \tilde{\lambda}_{2 i}, \tilde{\mu}_{2 i}$ and $\tilde{\varphi}_{i}$ are defined by equations in (10) for NICU-HDU and SCBU-TC, respectively.

The rejection probability at the $i$ th level of care is calculated as

$$
R_{i}=\sum_{\mathbf{n} \in T_{i}} \tilde{\pi}(\mathbf{n}) / \sum_{\mathbf{n} \in S} \tilde{\pi}(\mathbf{n}), \quad i=1,2,
$$

where $T_{1}=\left\{\mathbf{n} \in S \mid n_{1}=c_{1}\right\}$ and $T_{2}=\left\{\mathbf{n} \in S \mid n_{2}=c_{2}\right\}$.

\subsection{Model for a level 3/level 2 neonatal unit}

We derive the mathematical model for a level 3/level 2 neonatal unit as described in Section 2 and showing in Figure 4. Let $c_{1}, c_{2}$ and $c_{3}$ be the number of cots at NICU-HDU, SCBU and TC, respectively. Let $X_{i}(t)$ be the number of neonates at unit $i$, and $X_{i j}(t)$ be the number of neonates overflowing from unit $i$ to unit $j, i, j \in\{1,2,3\}$ at time $t$. Then the vector process

$$
\mathbf{X}=\left(X_{1}(t), X_{12}(t), X_{2}(t), X_{21}(t), X_{23}(t), t \geq 0\right)
$$

is a continuous-time discrete-valued stochastic process. We assume the process is time homogeneous, aperiodic and irreducible on its finite state space. The process does not necessarily need to hold the Markov property. The state space is given by

$$
S=\left\{\mathbf{n}=\left(n_{1}, o_{12}, n_{2}, o_{21}, o_{23}\right): n_{1}+o_{21} \leq c_{1}, o_{12}+n_{2} \leq c_{2}, o_{23} \leq c_{3}\right\},
$$

where $n_{i}, i=1,2$, is the number of neonates at the $i$ th main unit, and $o_{i j}, i, j \in\{1,2,3\}$, is the number of neonates at the $j$ th overflow unit from the $i$ th unit. Now the system can be modelled as two joint loss queueing processes with overflow. Assume that the joint GI/G/c/0 systems are in steady state. 
We shall now derive the expression for the steady state distribution for a level 3/level 2 neonatal unit. Extending the Theorem 1 for two joint GI/G/c/0 systems, the steady state distribution for a level 3 or level 2 neonatal unit with overflows can be derived.

Theorem 2. The steady state distribution for a level 3 or level 2 unit can be given by

$$
\pi^{a}(\mathbf{n})=\pi^{d}(\mathbf{n})=K^{-1} \prod_{i=0}^{\left(n_{1}+o_{21}-1\right)} \prod_{j=0}^{\left(n_{2}+o_{12}+o_{23}-1\right)} \frac{\lambda_{1 i}}{\mu_{1(i+1)}} \cdot \frac{\lambda_{2 j}}{\mu_{2(j+1)}},
$$

and

$$
\pi(\mathbf{n})=\pi^{a}(\mathbf{n}) \varphi_{\mathbf{n}}
$$

where $\lambda_{1 i}, \lambda_{2 j}, \mu_{1 i}, \mu_{2 j}, \varphi_{i}$ are arrival and departure related quantities for NICU-HDU and SCBU-TC, respectively, defined by equations in (6), and

$$
K=\sum_{\mathbf{n} \in S} \prod_{i=0}^{\left(n_{1}+o_{21}-1\right)} \prod_{j=0}^{\left(n_{2}+o_{12}+o_{23}-1\right)} \frac{\lambda_{1 i}}{\mu_{1(i+1)}} \cdot \frac{\lambda_{2 j}}{\mu_{2(j+1)}}
$$

is the normalising constant.

The approximate steady state distribution for a level 3/level 2 neonatal unit is given by

$$
\tilde{\pi}^{a}(\mathbf{n})=\tilde{\pi}^{d}(\mathbf{n})=\tilde{K}^{-1} \prod_{i=0}^{\left(n_{1}+o_{21}-1\right)\left(n_{2}+o_{12}+o_{23}-1\right)} \prod_{j=0}^{\tilde{\lambda}_{1 i}} \frac{\tilde{\lambda}_{2 j}}{\tilde{\mu}_{1(i+1)}},
$$

and

$$
\tilde{\pi}(\mathbf{n})=\tilde{\pi}^{a}(\mathbf{n}) \tilde{\varphi}_{\mathbf{n}}
$$

where $\tilde{\lambda}_{1 i}, \tilde{\mu}_{1 i}, \tilde{\lambda}_{2 i}, \tilde{\mu}_{2 i}$ and $\tilde{\varphi}_{i}$ are defined by equations in (10) for NICU-HDU and SCBU-TC, respectively, and

$$
\tilde{K}=\sum_{\mathbf{n} \in \mathbf{S}} \prod_{i=0}^{\left(n_{1}+o_{21}-1\right)} \prod_{j=0}^{\left(n_{2}+o_{12}+o_{23}-1\right)} \frac{\tilde{\lambda}_{1 i}}{\tilde{\mu}_{1(i+1)}} \cdot \frac{\tilde{\lambda}_{2 j}}{\tilde{\mu}_{2(j+1)}}
$$


The rejection probability at the $i$ th level of care for a level 3/level 2 neonatal unit is computed as

$$
R_{i}=\sum_{\mathbf{n} \in T_{i}} \tilde{\pi}(\mathbf{n}) / \sum_{\mathbf{n} \in S} \tilde{\pi}(\mathbf{n}),
$$

where

$$
T_{1}=\left\{\mathbf{n} \in S \mid\left(n_{1}+o_{21}=c_{1} \text { and } o_{12}+n_{2}=c_{2}\right)\right\}
$$

and

$$
T_{2}=\left\{\mathbf{n} \in S \mid\left(o_{12}+n_{2}=c_{2}, n_{1}+o_{21}=c_{1} \text { and } o_{23}=c_{3}\right)\right\} .
$$

The overflow probability $O_{i}, i=1,2$ at the $i$ th level of care for a level $3 /$ level 2 unit can also be computed from equation (11) substituting $T_{i}$ by $\left\{T_{i}^{*} \backslash T_{i}\right\}, i=1,2$,

where

$$
T_{1}^{*}=\left\{\mathbf{n} \in S \mid\left(n_{1}=c_{1} \text { and } o_{12}+n_{2}<c_{2}\right)\right\} \text {, }
$$

and

$$
\begin{aligned}
T_{2}^{*}=\left\{\mathbf{n} \in S \mid\left(n_{2}+o_{12}=\right.\right. & \left.c_{2} \text { and } n_{1}+o_{21}<c_{1}\right) \text { or } \\
& \left.\left(o_{12}+n_{2}=c_{2}, n_{1}+o_{21}=c_{1} \text { and } o_{23}<c_{3}\right)\right\} .
\end{aligned}
$$

Corollary 3. The approximate steady state distribution for a level 3 or level 2 neonatal unit is exact for exponential inter-arrival time and length of stay distributions at each level of care.

Proof. In the case of exponential inter-arrival time and length of stay distributions, arrival and departure related parameters reduce to the corresponding mean values of inter-arrival and length of stay

$$
m_{1 A, n}^{d}=q_{1 A}=m_{1 A}=\frac{1}{\lambda_{1}}, \quad m_{1 L, n}^{a}=m_{1 L, n}^{d}=q_{1 L}=m_{1 L}=\frac{1}{\mu_{1}}
$$




$$
m_{2 A, n}^{d}=q_{2 A}=m_{2 A}=\frac{1}{\lambda_{2}}, \quad m_{2 L, n}^{a}=m_{2 L, n}^{d}=q_{2 L}=m_{2 L}=\frac{1}{\mu_{2}}
$$

and

$$
\varphi_{\mathbf{n}}=1
$$

Then the steady state solution becomes

$$
\pi^{a}(\mathbf{n})=\pi^{d}(\mathbf{n})=K^{-1} \prod_{i=0}^{\left(n_{1}+o_{21}-1\right)} \frac{\lambda_{1}}{(i+1) \mu_{1}} \prod_{j=0}^{\left(n_{2}+o_{12}+o_{23}-1\right)} \frac{\lambda_{2}}{(j+1) \mu_{2}}
$$

Hence we obtain

$$
\pi(\mathbf{n})=K^{-1} \frac{\left(\frac{\lambda_{1}}{\mu_{1}}\right)^{\left(n_{1}+o_{21}\right)}\left(\frac{\lambda_{2}}{\mu_{2}}\right)^{\left(o_{12}+n_{2}+o_{23}\right)}}{\left(n_{1}+o_{21}\right) !\left(o_{12}+n_{2}+o_{23}\right) !}
$$

where

$$
K=\sum_{\mathbf{n} \in S} \frac{\left(\frac{\lambda_{1}}{\mu_{1}}\right)^{\left(n_{1}+o_{21}\right)}\left(\frac{\lambda_{2}}{\mu_{2}}\right)^{\left(o_{12}+n_{2}+o_{23}\right)}}{\left(n_{1}+o_{21}\right) !\left(o_{12}+n_{2}+o_{23}\right) !}
$$

which is the steady state solution for a level 3 unit as in Asaduzzaman and Chaussalet (2011) for Markovian arrival and discharge patterns. Adding back transfers, we can easily obtain the steady state distribution for a level 2 unit.

\section{Application of the model}

\subsection{The case study}

We apply the model to the case of a perinatal network in London which is the north central London perinatal network (NCLPN). The network consists of five neonatal units: UCLH (level 3), Barnet (level 2), Whittington (level 2), Royal Free (level 1 with ITU) and Chase Farm (level 1). The underlying 
Table 1: Inter-arrival and length of stay for the neonatal units in the NCLPN in 2008

\begin{tabular}{lcc}
\hline Unit & Mean inter-arrival & Mean length of stay \\
\hline UCLH & & \\
NICU-HDU & 0.58 & 11.51 \\
SCBU-TC & 0.24 & 5.83 \\
\hline Barnet & & \\
NICU-HDU & 1.12 & 6.78 \\
SCBU-TC & 0.83 & 9.71 \\
\hline Whittington & & 5.16 \\
NICU-HDU & 1.11 & 14.61 \\
SCBU-TC & 0.88 & \\
\hline Royal Free & & 2.21 \\
ITU & 2.77 & 9.99 \\
SCBU & 0.91 & 8.03 \\
\hline Chase Farm & & \\
SCBU & 1.05 & \\
\hline
\end{tabular}

aim of the network is to achieve capacity so that $95 \%$ women and neonates may be cared for within the network.

Data on admission and length of stay were provided by each of the units. Since the data did not contain the actual arrival rate and the rejection probability for the units we estimated the actual arrival rates using SIMUL8 ${ }^{\circledR}$ (SIMUL8, 2000), a computer simulation package designed to model and measure performance of a stochastic service system. Table 1 presents mean length of stay and estimated mean inter-arrival times for each level of care at UCLH, Barnet, Whittington, Royal Free and Chase Farm neonatal units for the year 
2008. Then we also use simulation (SIMUL8) to estimate the rejection probabilities for each level of care of the units for various arrival and discharge patterns. We refer to these estimates as 'observed' rejection probabilities.

\subsection{Numerical results and discussion}

In this section rejection probabilities are estimated for all five units in the NCLPN through the application of the model formulae in Section 3. An extensive numerical investigation has been carried out for a variety of interarrival and length of stay distributions to test the performance of the model and the approximation method. A common approach to check the accuracy of a generalised queueing model is to consider a variety of interarrival and LOS distributions and compare performance measures derived through the model with exact or simulated results (Chandy et al., 1975; Kerbachea and Smith, 1987; Altiok, 1989; Smith, 2003; Choi et al., 2005; Atkinson, 2009). In this study, exponential (M), two-phase hyper-exponential $\left(\mathrm{H}_{2}\right)$ and two-phase Erlang $\left(\mathrm{E}_{2}\right)$ distributions are considered to derive rejection probabilities as these were suggested to be appropriate interarrival and LOS distributions for the five neonatal hospitals we considered (Asaduzzaman et al., 2010a; Asaduzzaman, 2010). Hyper-exponential distributions were also found apprpriate by Griffiths et al. (2006) in the context of a classical ICU.

Table 2 compares the 'observed' and estimated rejection probabilities at each level of care for UCLH, Barnet, Whittington, Royal Free and Chase Farm neonatal units for various combinations of inter-arrival time and length

of stay distributions. Namely, exponential (M), two-phase hyper-exponential $\left(\mathrm{H}_{2}\right)$ and two-phase Erlang $\left(\mathrm{E}_{2}\right)$ distributions are considered. To compare 'observed' rejection probabilities with estimated rejection probabilities when 
Table 2: Comparison of rejection probabilities for different distributions at all five neonatal units in the NCLPN

\begin{tabular}{|c|c|c|c|c|}
\hline $\begin{array}{l}\text { UCLH } \\
\text { (17 NICU, } 12 \text { SCBU } \\
\text { and } 8 \text { TC cots })\end{array}$ & System notation & 'Observed' rej. prob. & Est. rej. prob. & Abs. per. err. \\
\hline NICU-HDU & $\mathrm{M} / \mathrm{M} / \mathrm{c} / 0$ & 0.1895 & 0.1962 & 3.54 \\
\hline SCBU-TC & & 0.1319 & 0.1271 & 3.64 \\
\hline NICU-HDU & $\mathrm{M} / \mathrm{H}_{2} / \mathrm{c} / 0$ & 0.1989 & 0.1933 & 2.82 \\
\hline SCBU-TC & & 0.1186 & 0.1313 & 10.71 \\
\hline NICU-HDU & $\mathrm{H}_{2} / \mathrm{M} / \mathrm{c} / 0$ & 0.2123 & 0.1706 & 19.64 \\
\hline SCBU-TC & & 0.1214 & 0.1010 & 16.80 \\
\hline NICU-HDU & $\mathrm{M} / \mathrm{E}_{2} / \mathrm{c} / 0$ & 0.2096 & 0.1987 & 5.20 \\
\hline SCBU-TC & & 0.1405 & 0.1235 & 12.10 \\
\hline NICU-HDU & $\mathrm{E}_{2} / \mathrm{M} / \mathrm{c} / 0$ & 0.2179 & 0.2347 & 7.71 \\
\hline SCBU-TC & & 0.1332 & 0.1652 & 24.02 \\
\hline NICU-HDU & $\mathrm{H}_{2} / \mathrm{H}_{2} / \mathrm{c} / 0$ & 0.1852 & 0.1669 & 9.88 \\
\hline SCBU-TC & & 0.1255 & 0.1077 & 14.18 \\
\hline NICU-HDU & $\mathrm{H}_{2} / \mathrm{E}_{2} / \mathrm{c} / 0$ & 0.1848 & 0.1726 & 4.98 \\
\hline SCBU-TC & & 0.0996 & 0.0970 & 2.61 \\
\hline NICU-HDU & $\mathrm{E}_{2} / \mathrm{H}_{2} / \mathrm{c} / 0$ & 0.2155 & 0.2332 & 8.21 \\
\hline SCBU-TC & & 0.1512 & 0.1672 & 10.58 \\
\hline NICU-HDU & $\mathrm{E}_{2} / \mathrm{E}_{2} / \mathrm{c} / 0$ & 0.2260 & 0.2367 & 4.73 \\
\hline SCBU-TC & & 0.1353 & 0.1626 & 20.18 \\
\hline
\end{tabular}

\section{Barnet}

(6 NICU, 14 SCBU

and $4 \mathrm{TC}$ cots)

\begin{tabular}{llllr}
\hline NICU-HDU & $\mathrm{M} / \mathrm{M} / \mathrm{c} / 0$ & 0.1644 & 0.1508 & 8.27 \\
SCBU-TC & & 0.0142 & 0.0076 & $*$ \\
\hline NICU-HDU & $\mathrm{M} / \mathrm{H}_{2} / \mathrm{c} / 0$ & 0.1496 & 0.1614 & 7.89 \\
SCBU-TC & & 0.0117 & 0.0111 & 7.23 \\
\hline NICU-HDU & $\mathrm{H}_{2} / \mathrm{M} / \mathrm{c} / 0$ & 0.1411 & 0.1513 & $*$ \\
SCBU-TC & & 0.0147 & 0.0097 & 13.31 \\
\hline NICU-HDU & $\mathrm{M} / \mathrm{E}_{2} / \mathrm{c} / 0$ & 0.1653 & 0.1433 & $*$ \\
SCBU-TC & & 0.0141 & 0.0051 & 15.31 \\
\hline NICU-HDU & $\mathrm{E}_{2} / \mathrm{M} / \mathrm{c} / 0$ & 0.1326 & 0.1529 & 0.95 \\
SCBU-TC & & 0.0055 & 0.0020 & $*$ \\
\hline NICU-HDU & $\mathrm{H}_{2} / \mathrm{H}_{2} / \mathrm{c} / 0$ & 0.1586 & 0.1571 & 2.32 \\
SCBU-TC & & 0.0125 & 0.0134 & $*$ \\
\hline NICU-HDU & $\mathrm{H}_{2} / \mathrm{E}_{2} / \mathrm{c} / 0$ & 0.1508 & 0.1473 & 3.61 \\
SCBU-TC & & 0.0142 & 0.0072 & $*$ \\
\hline NICU-HDU & $\mathrm{E}_{2} / \mathrm{H}_{2} / \mathrm{c} / 0$ & 0.1691 & 0.1752 & 6.78 \\
SCBU-TC & & 0.0034 & 0.0037 & $*$ \\
\hline NICU-HDU & $\mathrm{E}_{2} / \mathrm{E}_{2} / \mathrm{c} / 0$ & 0.1269 & 0.1355 & 0.0007 \\
SCBU-TC & & 0.0059 & &
\end{tabular}

${ }^{*}$ APEs are ignored for rejection probabilities $<0.05$ 
Continuation of Table 2

\begin{tabular}{|c|c|c|c|c|}
\hline $\begin{array}{l}\text { Whittington } \\
(12 \text { NICU, } 16 \text { SCBU } \\
\text { and } 5 \text { TC cots) }\end{array}$ & System notation & 'Observed' rej. prob. & Est. rej. prob. & Abs. per. err. \\
\hline NICU-HDU & $\mathrm{M} / \mathrm{M} / \mathrm{c} / 0$ & 0.0216 & 0.0007 & * \\
\hline SCBU-TC & & 0.0138 & 0.0018 & * \\
\hline NICU-HDU & $\mathrm{M} / \mathrm{H}_{2} / \mathrm{c} / 0$ & 0.0009 & 0.0026 & * \\
\hline SCBU-TC & & 0.0003 & 0.0128 & * \\
\hline NICU-HDU & $\mathrm{H}_{2} / \mathrm{M} / \mathrm{c} / 0$ & 0.0042 & 0.0000 & * \\
\hline SCBU-TC & $10^{2} / 20 / 0 / 0$ & 0.0110 & 0.0011 & * \\
\hline NICU-HDU & $\mathrm{M} / \mathrm{E}_{2} / \mathrm{c} / 0$ & 0.0097 & 0.0015 & * \\
\hline SCBU-TC & & 0.0029 & 0.0054 & * \\
\hline NICU-HDU & $\mathrm{E}_{2} / \mathrm{M} / \mathrm{c} / 0$ & 0.0006 & 0.0000 & * \\
\hline SCBU-TC & & 0.0010 & 0.0011 & * \\
\hline NICU-HDU & $\mathrm{H}_{2} / \mathrm{H}_{2} / \mathrm{c} / 0$ & 0.0053 & 0.0035 & * \\
\hline SCBU-TC & & 0.0091 & 0.0225 & * \\
\hline NICU-HDU & $\mathrm{H}_{2} / \mathrm{E}_{2} / \mathrm{c} / 0$ & 0.0002 & 0.0026 & * \\
\hline SCBU-TC & & 0.0236 & 0.0134 & * \\
\hline NICU-HDU & $\mathrm{E}_{2} / \mathrm{H}_{2} / \mathrm{c} / 0$ & 0.0003 & 0.0000 & * \\
\hline SCBU-TC & & 0.0002 & 0.0024 & * \\
\hline NICU-HDU & $\mathrm{E}_{2} / \mathrm{E}_{2} / \mathrm{c} / 0$ & 0.0018 & 0.0000 & * \\
\hline SCBU-TC & & 0.0005 & 0.0005 & * \\
\hline \multicolumn{5}{|c|}{$\begin{array}{l}\text { Royal Free } \\
(2 \text { ITU and } 12 \text { SCBU cots })\end{array}$} \\
\hline ITU & $\mathrm{M} / \mathrm{M} / \mathrm{c} / 0$ & 0.1468 & 0.1504 & 2.45 \\
\hline SCBU & & 0.1558 & 0.1580 & 1.41 \\
\hline ITU & $\mathrm{M} / \mathrm{H}_{2} / \mathrm{c} / 0$ & 0.1714 & 0.1504 & 12.25 \\
\hline SCBU & & 0.1476 & 0.1580 & 7.05 \\
\hline ITU & $\mathrm{H}_{2} / \mathrm{M} / \mathrm{c} / 0$ & 0.1667 & 0.1556 & 6.66 \\
\hline $\mathrm{SCBU}$ & & 0.1509 & 0.1476 & 2.19 \\
\hline ITU & $\mathrm{M} / \mathrm{E}_{2} / \mathrm{c} / 0$ & 0.1560 & 0.1504 & 3.59 \\
\hline SCBU & & 0.1393 & 0.1580 & 13.42 \\
\hline ITU & $\mathrm{E}_{2} / \mathrm{M} / \mathrm{c} / 0$ & 0.1756 & 0.1504 & 14.35 \\
\hline SCBU & & 0.1516 & 0.1685 & 11.15 \\
\hline ITU & $\mathrm{H}_{2} / \mathrm{H}_{2} / \mathrm{c} / 0$ & 0.1681 & 0.1351 & 19.63 \\
\hline SCBU & & 0.1452 & 0.1476 & 1.65 \\
\hline ITU & $\mathrm{H}_{2} / \mathrm{E}_{2} / \mathrm{c} / 0$ & 0.1481 & 0.1556 & 5.06 \\
\hline SCBU & & 0.1680 & 0.1476 & 12.14 \\
\hline ITU & $\mathrm{E}_{2} / \mathrm{H}_{2} / \mathrm{c} / 0$ & 0.1252 & 0.1347 & 7.59 \\
\hline SCBU & & 0.1384 & 0.1685 & 21.75 \\
\hline ITU & $\mathrm{E}_{2} / \mathrm{E}_{2} / \mathrm{c} / 0$ & 0.1315 & 0.1579 & 20.08 \\
\hline $\mathrm{SCBU}$ & & 0.1619 & 0.1685 & 4.08 \\
\hline
\end{tabular}




\begin{tabular}{|c|c|c|c|c|}
\hline $\begin{array}{l}\text { Chase Farm } \\
\text { (10 SCBU cots) }\end{array}$ & System notation & 'Observed' rej. prob. & Est. rej. prob. & Abs. per. err. \\
\hline SCBU & $\mathrm{M} / \mathrm{M} / \mathrm{c} / 0$ & 0.1078 & 0.1060 & 1.67 \\
\hline SCBU & $\mathrm{M} / \mathrm{H}_{2} / \mathrm{c} / 0$ & 0.1094 & 0.1060 & 3.11 \\
\hline $\mathrm{SCBU}$ & $\mathrm{H}_{2} / \mathrm{M} / \mathrm{c} / 0$ & 0.1474 & 0.1233 & 16.35 \\
\hline $\mathrm{SCBU}$ & $\mathrm{M} / \mathrm{E}_{2} / \mathrm{c} / 0$ & 0.1047 & 0.1060 & 1.24 \\
\hline SCBU & $\mathrm{E}_{2} / \mathrm{M} / \mathrm{c} / 0$ & 0.0719 & 0.0792 & 10.15 \\
\hline SCBU & $\mathrm{H}_{2} / \mathrm{H}_{2} / \mathrm{c} / 0$ & 0.1418 & 0.1233 & 13.0 \\
\hline SCBU & $\mathrm{H}_{2} / \mathrm{E}_{2} / \mathrm{c} / 0$ & 0.1469 & 0.1233 & 16.0 \\
\hline SCBU & $\mathrm{E}_{2} / \mathrm{H}_{2} / \mathrm{c} / 0$ & 0.0817 & 0.0792 & 3.06 \\
\hline SCBU & $\mathrm{E}_{2} / \mathrm{E}_{2} / \mathrm{c} / 0$ & 0.0700 & 0.0792 & 13.14 \\
\hline
\end{tabular}

one of these probabilities are 0.05 or more, we define 'absolute percentage error' (APE) as the absolute deviation between 'observed' and estimated rejection probability divided by 'observed' rejection probability and then multiplied by 100. Rejection probabilities below 0.05 are normally considered satisfactory. For this reason we have not reported the APE when both 'observed' and estimated rejection probabilities are less than 0.05.

The 'observed' and estimated rejection probabilities are close for the UCLH unit. At NICU-HDU, the highest 'observed' rejection probability is occurred for $\mathrm{E}_{2} / \mathrm{E}_{2} / \mathrm{c} / 0$, and the estimated rejected probability is also highest for the same arrival and discharge patterns with an absolute percentage error (APE) 4.73\%. The lowest 'observed' rejection probability is 0.1848 for the $\mathrm{H}_{2} / \mathrm{E}_{2} / \mathrm{c} / 0$ while the estimated rejection probability is 0.1726 with an $\mathrm{APE} 4.98 \%$. At SCBU for $\mathrm{E}_{2} / \mathrm{M} / \mathrm{c} / 0$, the 'observed' and estimated rejection probabilities are 0.1332 and 0.1652 , respectively, with an APE 24.02\%. At Barnet NICU-HDU, the 'observed' and estimated rejection probabilities are close with a varying APEs from 0.95\%-15.31\%. For Barnet SCBU the 'observed' and estimated rejection probabilities are all less than 0.05 and 
relatively close to each other. Both the UCLH NICU-HDU and SCBU and Barnet NICU-HDU would require additional cots to keep the rejection level low and achieve a 0.05 target.

Rejection probabilities from both NICU-HDU and SCBU at the Whittington neonatal unit are below 0.05 regardless of the combination of inter-arrival time and length of stay distributions, which indicates that the neonatal unit is performing well with 12 NICU, 16 SCBU and 5 TC cots. The 'observed' and estimated rejection probabilities at Royal Free ITU and SCBU and Chase Farm SCBU are close to each other. The results in Table 2 suggest that Royal Free ITU and SCBU and Chase Farm SCBU require extra cots to decrease the rejection level.

Through our extensive numerical investigations we observe that the rejection probability often varies greatly according to arrival and discharge patterns. The number of cots required will also vary depending upon arrival and discharge patterns. Therefore, one should take into account the actual arrival and discharge patterns for accurate capacity planning of neonatal units rather than approximating by Markovian arrival and discharge patterns. To achieve a '95\%' admission acceptance target UCLH NICU-HDU and SCBU, Barnet NICU-HDU, Royal Free ITU and SCBU, and Chase Farm SCBU need to increase their number of cots.

We have also observed that performance of the proposed generalised capacity planning model improves as the squared coefficient of variation values of inter-arrival and length of stay get closer to 1 (recall that our approximation is exact for the Markovian inter-arrival and length of stay case in which squared coefficient of variation values of inter-arrival and length of stay are 
both 1) and as $\lambda / \mu$ gets larger (i.e., under heavy traffic). A possible explanation is that as $\lambda / \mu$ gets larger, the period during which all the cots are busy tends to get longer. As such a busy period gets longer, arrival and departure points of arrivals tend to become more and more like arbitrary points in time. As such, the approximation is likely to get more accurate.

\section{Conclusion}

Planning capacity accurately has been an important issue in the neonatal sector because of the high cost of care, in particular. Markovian arrival and length of stay can provide only approximate estimates which may often underestimate or overestimate the required capacity. The underestimation of cots may increase the rejection level, which in turn may be life-threatening or cause expensive transfers for high risk neonates, hence increase risk for vulnerable babies. On the other hand, overestimation may cause underutilisation of cots, and potential waste of resources.

In this paper a generalised framework for determining cot capacity of a perinatal network was derived. After decomposing the whole network into neonatal units, each unit was analysed separately. Expressions for the stationary distribution and for rejection probabilities were derived for each neonatal unit. An approximation method was suggested to obtain the steady state rejection probabilities. The model formulation was then applied to the neonatal units in the NCLPN. A variety of inter-arrival and length of stay distributions in the neonatal units has been considered for numerical experimentation. The 'observed' and estimated rejection probabilities were close (APE typically less than 20\%) for all hospital units when rejection proba- 
bilities were 0.05 or more. When 'observed' rejection probabilities were less than 0.05, as for the Barnet SCBU and both the Whittington NICU-HDU and SCBU, the APE increased rapidly to beyond $50 \%$. However, since these values are less than or close to 0.05 , they do not have an impact on management decisions regarding the number of cots. In contrast, when 'observed' rejection probabilities are high, then the estimated values become close to each other. The 'observed' and estimated rejection probabilities were, in general, close for high traffic intensities. As traffic intensity drops the absolute percent error increases quickly. In most cases, the absolute percent error becomes small for Markovian arrival and length of stay patterns. We know that service time distribution is insensitive for delay systems if the arrival process is Poisson. However, the property is no longer valid for loss systems. The model results as seen in Table 2 also confirm this sensitivity property.

The main advantage of the model framework is that arrival and discharge pattern do not need to hold the Markov property. The model is based on the first two moments and requires no distributional assumption. This twomoment approximation techniques performs reasonably well in terms of accuracy (APE) and is fast. The method is exactly Markovian for equal mean and variance. The numerical results show that the model can be used as a capacity planning tool for perinatal networks for non-Markovian arrival and discharge patterns as well as Markovian patterns. If good estimates of the first two moments are available, then the generalised model can be used to determine the required cot capacity in a perinatal network for given level of rejection probabilities. 


\section{References}

Altiok, T., 1989. Approximate analysis of queues in series with phase-type service times and blocking, Operations Research 37, 601-610.

Asaduzzaman, M., Chaussalet, T. J., 2008. Modelling and performance measure of a perinatal network centre in the United Kingdom, In proceedings of the 21st IEEE International Symposium on Computer-Based Medical Systems, 506-511.

Asaduzzaman, M., Chaussalet, T. J., Robertson, N. J., 2010a. A loss network model with overflow for capacity planning of a neonatal unit, Annals of Operations Research 178, 67-76.

Asaduzzaman, M., 2010. Capacity planning of a perinatal network: a loss network framework, $\mathrm{PhD}$ Thesis, University of Westminster.

Asaduzzaman, M., T. J. Chaussalet, S. Adeyemi, S. Chahed, J. Hawdon, D. Wood, N. J. Robertson. 2010b. Towards effective capacity planning in a perinatal network centre, Archives of Disease in Childhood 95, F283-F287.

Asaduzzaman, M., Chaussalet, T. J., 2011. An overflow loss network model for capacity planning of a perinatal network, Journal of the Royal Statistical Society: Series A (Statistics in Society) 174, 403-417.

Atkinson, J. B., 2008. Two new heuristics for the GI/G/n/0 queueing loss system with examples based on the two-phase Coxian distribution, Journal of the Operational Research Society 60, 818-830. 
Bliss, 2007. Too Little, Too Late?, Bliss - The Premature Baby Charity, London. Available at http://www.bliss.org.uk/wp-content/uploads/2011/11/too_little_too_late_web.pdf (accessed on June 24, 2013).

Brandt, A., Lisek, B., 1980. On the approximation of GI/GI/m/0 by means of GI/GI/ $\infty$, Journal of Information Processing and Cybernetics 16, 597600 .

Chandy, K., M., Herzog, U., Woo, L., 1975. Approximate analysis of general queuing networks, IBM Journal of Research and Development 19, 43-49.

Choi, D. W., Kim, N. M., Chae, K. C., 2005. A two-moment approximation for the GI/G/c queue with finite capacity, INFORMS Journal on Computing $17,75-81$.

Franken, P., Konig, D., Arndt, U., Schmidt, V., 1982. Queues and Point Processes, Chichester: Wiley.

Griffiths, J. D., Price-Lloyds, N., Smithies, M. Williams, J., 2006. A queueing model of activities in an intensive care unit, IMA Journal of Management Mathematics 17, 277-288.

Hsin, W. J., Van de Liefvoort, A., 1996. The teletraffic analysis of the multiserver loss model with renewal distributions, Telecommunication Systems $5,303-321$.

Izady, N., Worthington, D., 2011. Approximate analysis of non-stationary loss queues and networks of loss queues with general service time distributions, European Journal of Operational Research 213, 498-508. 
Kelly, F. P., 1979. Reversibility and Stochastic Networks, Chichester: Wiley.

Kelly, F.P., 1991. Loss networks, Annals of Applied Probability 1, 319-378.

Kerbachea, L., and Smith, J., M., 1987. The generalized expansion method for open finite queueing networks, European Journal of Operational Research 32, 448-461.

Kim, N. K., Chae, K. C., 2003. Transform-free analysis of the GI/G/1/K queue through the decomposed Little's formula, Computer \& Operations Research 30, 353-365.

Kimura, T., 2000. Equivalence relations in the approximations for the M/G/s/s+r queue, Mathematical and Computer Modelling 31, 215-224.

Kimura, T., 2003. A consistent diffusion approximation for finite-capacity multiserver queues, Mathematical and Computer Modelling 38, 1313-1324.

Klimenok, V., C. S. Kim, D. Orlovsky, A. Dudin. 2005. Lack of invariant property of the Erlang loss model in case of MAP input, Queueing Systems 49, 187-213.

Litvak, N., Van Rijsbergen, M., Boucherie, R. J., Van Houdenhoven, M., 2008. Managing the overflow of intensive care patients, European Journal of Operational Research 185, 998-1010.

Miyazawa, M., Tijms, H. C., 1993. Comparison of two approximations for the loss probability in finite-buffer queues, Probability in the Engineering and Informational Sciences 7, 19-27. 
National Audit Office, 2007. Caring for Vulnerable Babies: The Reorganisation of Neonatal Services in England, National Audit Office, London. Available at http: //www .nao.org.uk/wp-content/uploads/2007/12/0708101.pdf (accessed on June 24, 2013).

Rapoport, J., Teres, D., Zhao, Y., Lemeshow, S., 2003. Length of stay data as a guide to hospital economic performance for ICU patients, Medical Care 41, 386-397.

SIMUL8, 2000. SIMUL8 Manual and Simulation Guide, Glasgow: Visual Thinking International Limited.

Smith, J., M., 2003. M/G/c/K blocking probability models and system performance, Performance Evaluation 52, 237-267.

Takács, L., 1956. On the generalization of Erlang's formula, Acta Mathematica Hungarica 7, 419-433.

Takács, L., 1962. Introduction to the Theory of Queues, New York: Oxford University Press.

Van Dijk, N. M., 1993. Queueing Networks and Product Forms: A Systems Approach, Chichester: Wiley.

Van Dijk, N. M., N. Kortbeek, 2009. Erlang loss bounds for OT-ICU systems, Queueing Systems 63, 253-280.

Vasilakis, C., Marshall, A. H., 2005. Modelling nationwide hospital length of 
stay: opening the black box, Journal of the Operational Research Society $56,862-869$.

Weissman, C., 1997. Analyzing intensive care unit length of stay data: problems and possible solutions, Critical Care Medicine 25, 1594-1600.

Weissman, C., 2000. Analyzing the impact of long-term patients on ICU bed utilization, Intensive Care Medicine 26, 1319-1325.

Whitt, W., 1984. Heavy-traffic approximations for service systems with blocking, AT\&T Bell Lab Technical Journal 63, 689-708.

Whitt, W., 2004. A diffusion approximation for the G/GI/n/m queue, Operations Research 52, 922-941. 